\title{
Fast Identification of Transonic Buffet Envelope using Computational Fluid Dynamics
}

\section{Abstract}

Purpose - The paper presented a numerical method based on computational fluid dynamics that allows investigating the buffet envelope of reference equivalent wings at the equivalent cost of several two-dimensional, unsteady, turbulent flow analyses. The method bridges the gap between semi-empirical relations, generally dominant in the early phases of aircraft design, and three-dimensional turbulent flow analyses, characterised by high costs in analysis setups and prohibitive computing times.

Design/methodology/approach - Accuracy in the predictions and efficiency in the solution are two key aspects. Accuracy is maintained by solving a specialised form of the Reynolds-averaged Navier-Stokes equations valid for infinite-swept wing flows. Efficiency of the solution is reached by a novel implementation of the flow solver, as well as by combining solutions of different fidelity spatially.

Findings -Discovering the buffet envelope of a set of reference equivalent wings is accompanied with an estimate of the uncertainties in the numerical predictions. Just over 2,000 CPU hours are needed if it is admissible to deal with an uncertainty of $\pm 1.0 \mathrm{deg}$ in the angle of attack at which buffet onset/offset occurs. Halving the uncertainty requires significantly more computing resources, close to a factor 200 compared with the larger uncertainty case.

Practical implications - To permit the use of the proposed method as a practical design tool in the conceptual/preliminary aircraft design phases, the method offers the designer with the ability to gauge the sensitivity of buffet on primary design variables, such as wing sweep angle and chord to thickness ratio.

Originality/value - The infinite-swept wing, unsteady Reynolds-averaged Navier-Stokes equations have been successfully applied, for the first time, to identify buffeting conditions. This demonstrates the adequateness of the proposed method in the conceptual/preliminary aircraft design phases.

Keywords Buffet envelope, reference equivalent wings, computational fluid dynamics, infinite-swept wing, uncertainty.

Paper type Research paper

\section{Introduction}

The importance of buffet as a primary constraint in establishing the low and high-speed performance capabilities of transport aircraft cannot be underestimated. The reason is that the physical phenomenon of buffet originates from separated airflow that imposes an excitation on a structure, with the subsequent vibrations attributable to the dynamic response of that structure. These vibrations can have a strong impact on the aircraft aerodynamic and manoeuvrability capabilities, on the pilot's workload, 
and can cause structural damage and, potentially, catastrophic failure (Badcock et al., 2011). Traditionally, the buffet onset at $1.0 \mathrm{~g}$ condition is identified by flight tests as the speed at which the vibration reaches $\pm 0.050 \mathrm{~g}$ while windup turns are executed at constant Mach number (Bérard and Isikveren, 2009). For a given wing geometry, the $1.0 \mathrm{~g}$ buffet onset curve relates the lift coefficient at the onset of $1.0 \mathrm{~g}$ buffet, $C_{L B}$, with Mach number, $M$, implicitly showing the dependence on the angle of attack. Due to the inherent complexities and cost in carrying out flight testing, let alone being an unrealistic option in conceptual design, researchers have focussed on wind tunnel measurements and computational models to gain insights on buffet physical mechanisms and to develop appropriate methods for its prediction.

The experimental study (Benoit and Legrain, 1987) on RA16SC1 aerofoil and rectangular wing found that buffeting appears when the boundary-layer separation takes place at the shock region. Under certain operating conditions, a large amplitude and periodic motion of the shock may initiate, leading to oscillations of the entire flow-field. The three-dimensional (3D) buffet phenomenon was investigated in the work of (Dandois, 2016) for the half model of a wing-body configuration for several Mach and Reynolds numbers. The wing sweep angle, combined with the wing finiteness, was found to have a strong impact on buffet characteristics. While two-dimensional (2D) buffet exhibits a marked peak in the pressure spectra, the 3D buffet contains a broadband frequency content at higher Strouhal numbers. Unfortunately, wind tunnel measurements are not error-free. Among others, the reasons are that wind tunnel results need to be extrapolated to full scale for realistic Reynolds number flows, and the buffet onset may be influenced by the level of turbulence and flow unsteadiness in the wind tunnel facility.

The first numerical studies on RA16SC1 aerofoil (Girodroux-Lavigne and Le Balleur, 1987) and (Girodroux-Lavigne and Le Balleur, 1988) used a viscous, pseudo-inviscid, and interaction solver. The interaction step was performed by means of a semiimplicit scheme and convergence was achieved at each time step. The error between the real and pseudo-inviscid flow was obtained from the viscous solver, approximated by integral equations and solved by a marching scheme. A comprehensive review on shock buffet (Lee, 2001) references the two previously mentioned studies, and explains physical models of the shockbuffet mechanism. The study (Coustols et al. 2003) attempted to predict shock induced oscillations that appear over RA16SC1 aerofoil with the 2D unsteady Reynolds-averaged Navier-Stokes (URANS) solver, using one- and two-equation turbulence models. Later, the authors (Thiery and Coustols, 2005) addressed the same problem in more depth. Both studies pointed out that there is a great sensitivity of the results on both turbulence models and grid strategy. Nevertheless, a reasonable agreement between numerical and experimental data was observed for most of the turbulence models. More recently, other numerical studies on RA16SC1 aerofoil appeared. The study (Zhong et al., 2009) assessed fidelity of the solution of URANS, detached-eddy simulations (DES) and implicit large-eddy simulations (ILES) Navier-Stokes equations. The authors reported a good agreement of the computed pressure coefficient distribution with experimental data for all simulation methods. Nevertheless, ILES and DES managed to predict the flow-field more accurately than URANS. The work in (Iovnovich and Raveh, 2012) presented URANS simulations of the shock buffet phenomenon and looked at the characteristics of the shock-buffet instability mechanism for 
several aerofoils, including RA16SC1. The suitability of several turbulence models for shock-buffet analysis was also assessed. It was concluded that a good prediction of the buffet onset conditions and fair prediction of the buffet frequencies may be achieved using URANS simulations. The follow up study (Iovnovich and Raveh, 2015) looked at finite-wing configurations, based on the RA16SC1 aerofoil, at shock-buffet flow conditions. The effect of increasing the sweep angle on buffet characteristics was found similar to that of increasing the angle of attack, resulting in a decrease of the amplitude and an increase of the buffeting frequency. Their study of moderate aspect ratio, swept wing configurations revealed an interaction between the lateral propagating buffet cells and the wingtip vortices. For low aspect ratio wings, the tip phenomenon dominates the flow-field, and the shock remains steady state except for the tip region. For increasing aspect ratio, the effect of wing tip decreases and becomes limited to the tip region, whereas lateral propagation of buffet cells is observed across the wing span.

The purpose of this work is to present a method based on computational fluid dynamics (CFD) that provides, at the equivalent cost of 2D URANS runs, a fast identification of the buffet envelope of a finite-span, swept and straight wing, and the sensitivity of buffet when the geometry of the wing is modified. To permit the use of the proposed method as a practical design tool in the conceptual/preliminary design phases, the method offers the ability for any designer to gauge the sensitivity of buffet on primary design drivers, such as wing sweep angle and chord to thickness ratio. The proposed method bridges the gap between semi-empirical approaches (Bérard and Isikveren, 2009) and expensive 3D CFD analyses (Iovnovich and Raveh, 2015) in an attempt to bring physics-based methods earlier in the design process. The work is built around four technical objectives. The first objective is related to the validation of the present flow solver and numerical settings by comparison with experimental data of the RA16SC1 aerofoil. The second objective demonstrates the deployment of an efficient infinite-swept wing flow solver (2D grid stencil), recently developed by the authors (Franciolini et al., 2017), for the identification of the buffet envelope. The third objective addresses the challenge of improving the accuracy of the buffet envelope with higher fidelity (3D URANS) at a minimal additional cost. The last objective investigates the advantages of the proposed combined approach.

The paper continues in Section 2 with a short overview of the CFD solver. Section 3 describes the test cases. Section 4 collects the computed results and addresses the four technical objectives. Then, lessons learnt about numerical settings and grid best practice are given. Finally, conclusions are presented.

\section{Methods}

The flow solver employed in this study is DLR-Tau (Schwamborn et al., 2006), a finite volume based CFD flow solver used by several major aerospace industries across Europe. The DLR-Tau solver uses an edge-based vertex-centred scheme. The convective fluxes are discretized using several first- and second-order numerical schemes, including central and upwind types. 
The viscous fluxes are discretized using a second-order central scheme. Time integration is performed with explicit RungeKutta scheme or the Lower-Upper Symmetric Gauss-Seidel (LU-SGS) implicit approximate factorization scheme. Jameson's dual time stepping approach (Jameson et al., 2006) is employed for time-accurate computations. Convergence rate is accelerated using local-time stepping, implicit residual smoothing and multi-grid approach based on agglomerated coarse grids. Several models for turbulence closure are available including the one-equation Spalart-Allmaras (SA) type and more complex twoequation models of the $k-\omega$ family.

The flow solver also contains a very efficient algorithm for solving the steady and unsteady, incompressible and compressible flow around an infinite-swept wing. This specialised algorithm was implemented by the authors in DLR-Tau (Drofelnik and Da Ronch, 2017) and is now being used for production. More details about the algorithm, boundary conditions, and application on a variety of test cases are found in (Franciolini et al., 2017). The infinite-swept wing solver is a key capability for the work herein presented, and this paper may well be the first work demonstrating the use of the solver for buffeting flows around wing planforms commonly used in preliminary/conceptual design phases.

\section{Test cases}

The first test case is for the RA16SC1 supercritical aerofoil. The second test case is for an equivalent reference wing, as currently used by industry at the conceptual design phase (Bérard and Isikveren, 2009). The equivalent reference wing is a representation of an actual cranked wing by a simplified planform with a reduced number of primary design variables. Five wings are derived from the baseline wing by changing the sweep angle, with aspect ratio 10 and the RA16SC1 aerofoil as cross-section.

\section{RA16SC1 aerofoil}

Experimental measurements for the RA16SC1 supercritical aerofoil (Benoit and Legrain, 1987) were collected at Mach number $M_{\infty}=0.732$, temperature $T_{\infty}=283 \mathrm{~K}$, and Reynolds number $R e=4.2 \times 10^{6}$ for various angles of attack. The transition in the experiment was tripped on both sides of the aerofoil at $x / c=7.5 \%$. Strong shock oscillations were observed experimentally at $\alpha=3^{\circ}$. In simulations, though, corrections to Mach number and angle of attack are commonly used, as practised in (Thiery and Coustols, 2015). The suggestion is to correct Mach number and angle of attack by $M_{\infty}^{\prime}=M_{\infty}-0.009$ and $\alpha^{\prime}=\alpha-1 \mathrm{deg}$, respectively, where the prime superscript indicates the values used in the simulation. Other interest quantities (temperature, Reynolds number, and the transition location) are set to the nominal values of the experimental campaign.

The computational grid adopted for the flow simulations is shown in Figure 1. The grid was carefully designed based on previous suggestions and results (Coustols et al. 2003) and (Thiery and Coustols, 2015). The unstructured grid consists of about 27 
thousand mesh elements with wall refinement. The first layer on the wall was placed at $4 \times 10^{-6}$ (for a chord of one), ensuring $y^{+}<1$. The grid has 228 intervals along the aerofoil and the far-field boundary is placed at 50 chords from the aerofoil. For all calculations, the explicit time stepping and the fourth order Runge-Kutta scheme were used. For time-accurate calculations, Jameson's dual time stepping approach (Jameson et al., 2006) is employed. To accelerate the convergence to a steady state at each physical time-step, a local time-stepping, implicit residual smoothing and full multigrid acceleration techniques were used. The discretisation of the convective and diffusive fluxes of RANS/URANS equations is based on the second order Roe's flux difference splitting scheme, whereas the first order Roe scheme was used for the SA equation. Venkatakrishnan's flux limiter was used for all simulations reported in this paper. For all test cases, the original SA turbulence model (Spalart and Allmaras, 1994) was employed.

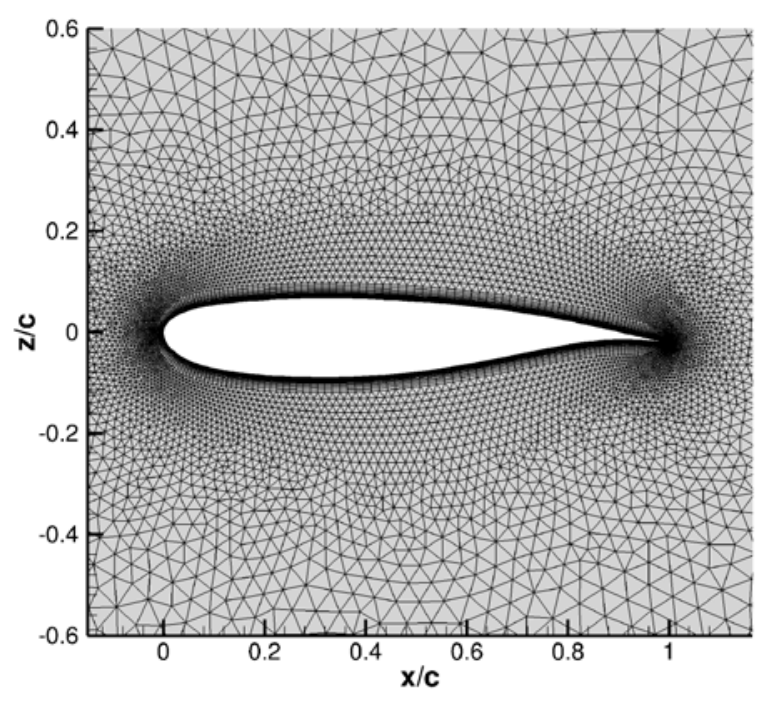

Figure 1 Unstructured grid of RA16SC1 supercritical aerofoil

A no-slip boundary condition was applied on the aerofoil surface, and far-field boundary conditions were applied at the farfield boundaries. The CFL number was set to 1.5 and the number of multigrid levels to 3 . Each simulation was first computed with the steady solver. The time-accurate computations were then restarted from the steady state solution. Time convergence study revealed that a non-dimensional time step $\Delta \bar{t}=a_{\infty} \Delta t / c=0.04$ is sufficient to achieve a solution independent from the temporal discretization, where $a_{\infty}$ represents the speed of sound, $\Delta t$ is the dimensional time step and $c$ is the aerofoil chord. The temporal refinement was conducted using four different time steps, $\Delta \bar{t}=a_{\infty} \Delta t / c=0.02,0.04,0.08$ and 0.16 (where $a_{\infty}$ represents the speed of sound, $\Delta t$ is the dimensional time step and $c$ is the aerofoil chord). The time convergence study revealed that a non-dimensional time step $\Delta \bar{t}=0.04$ is sufficient to achieve a solution independent from the temporal discretization.

\section{Reference equivalent wing}


The baseline model of the reference equivalent wing is un-swept, un-tapered with an aspect ratio of 10 . The wing cross-section is based on the RA16SC1 aerofoil. Five reference equivalent wings are derived from the baseline model by modifying the sweep angle, $\Lambda=10,20,30,40$ and $50 \mathrm{deg}$. The derived wings are built by stacking the 2D unstructured grid, Figure 1, in the span-wise direction from the mid-span symmetry plane to the lateral far-field boundary. The far-field boundary is located at 50 chords from the symmetry plane. For the grid generation, constant span-wise spacing of $4 \times 10^{-2}$ (for a chord of one) was used from mid-span to $90 \%$ semi-span, and grid points were clustered towards the tip. For all reference equivalent wings, 321 points were used in the span-wise direction. The grids contain about 6 million mesh elements, and a symmetry boundary condition at midspan was used to halve the computational cost. A no-slip boundary condition was applied on the wing surface, and far-field boundary conditions were applied at the far-field boundaries. Two representative grids are shown in Figure 2. The same numerical settings as for the RA16SC1 aerofoil were used.

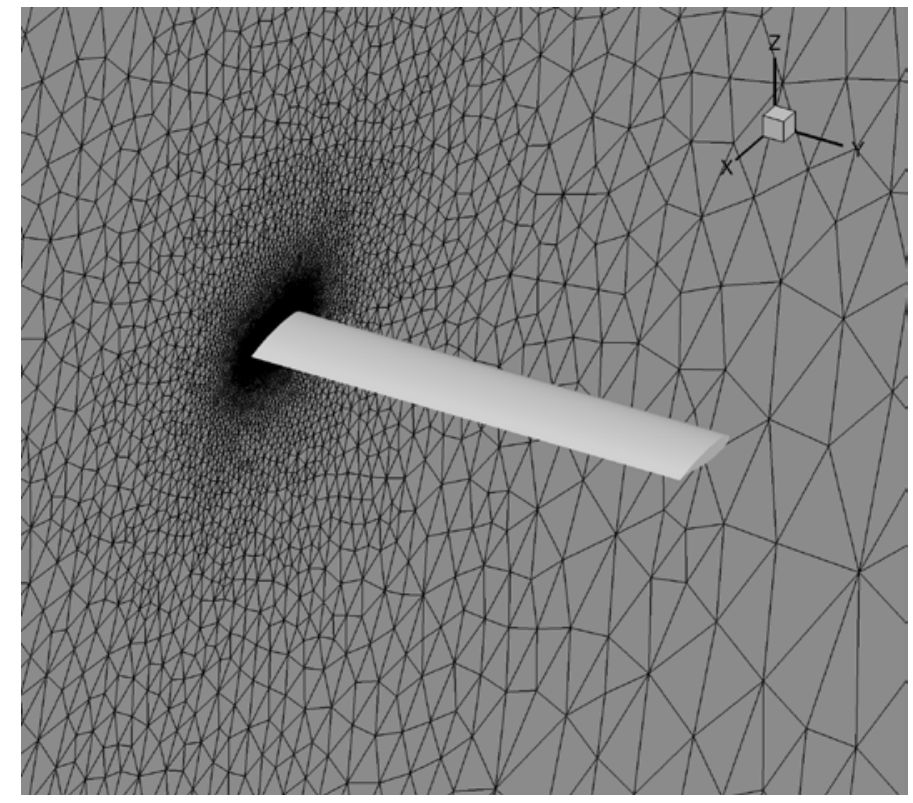

(a) Un-swept wing

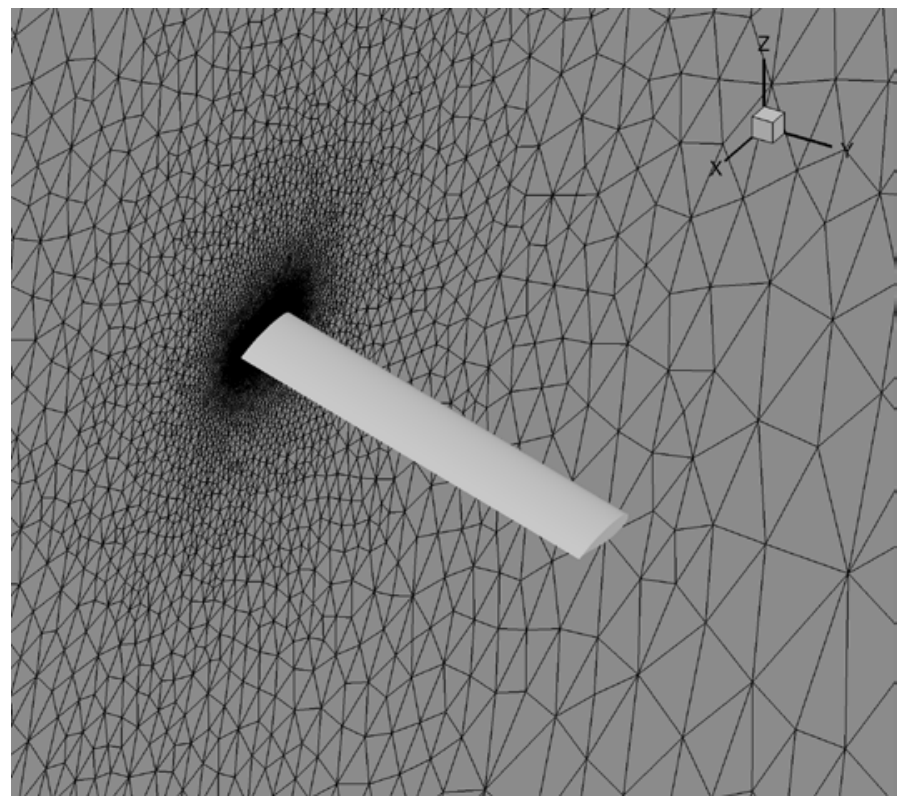

(b) 20 deg swept wing

Figure 2 Unstructured grids of the reference equivalent wings

\section{Results}

\section{Validation of RA16SC1 aerofoil}

First, 2D flow analyses were run solving the URANS equations coupled with SA turbulence model. The corrected flow conditions are for $M_{\infty}^{\prime}=0.723$ (uncorrected experimental value: $M_{\infty}=0.732$ ) and $\alpha^{\prime}=2.0,2.5,3.0$ and 3.5 deg (uncorrected experimental values: $\alpha=\alpha^{\prime}+1 \mathrm{deg}$ ). 
Table 1 RA16SC1 aerofoil shock-buffet: reduced frequency, $\bar{f}$, and lift coefficient amplitude, $\Delta C_{l}$, for various angles of attack $\left(M_{\infty}=0.732\right.$ and $\left.R e=4.2 \times 10^{6}\right)$; experimental data from (Benoit and Legrain, 1987).

\begin{tabular}{lllll}
\hline$\alpha(\mathrm{deg})$ & & $\bar{f}$ & \multicolumn{2}{c}{$\Delta C_{l}$} \\
& Exp. Data & CFD & Exp. Data & CFD \\
\hline 3.0 & 0.41 & 0.46 & 0.11 & 0.10 \\
3.5 & 0.43 & 0.40 & 0.25 & 0.35 \\
4.0 & 0.46 & 0.46 & 0.31 & 0.44 \\
4.5 & 0.50 & 0.45 & 0.26 & 0.53 \\
\hline
\end{tabular}

Table 1 reports the reduced frequency, $\bar{f}$, and the lift coefficient amplitude, $\Delta C_{l}$, for shock-buffet at four angles of attack. The reduced frequency of buffeting is defined as $\bar{f}=2 \pi f \mathrm{c} / U_{\infty}$, where $f$ is the dimensional shock-buffet frequency and $U_{\infty}$ is the dimensional freestream velocity. Qualitatively, the agreement between the two sources of data is good, with numerical predictions differing up to 6\% from experimental measurements. Quantitatively, experimental values of reduced frequency increase monotonically for increasing angle of attack, whereas the trend of the lift coefficient amplitude is concave with the angle of attack. Deviations between the experimental and numerical trends are consistent with other references (Thiery and Coustols, 2005).

Figure 3 reports the levels of root mean square constant head pressure fluctuations, $p_{r m s} / 0.5 \rho_{\infty} U_{\infty}$, along the aerofoil surface, where $\rho_{\infty}$ denotes the freestream fluid density. Note that $\mathrm{x} / \mathrm{c}<0$ is for the lower side and $\mathrm{x} / \mathrm{c}>0$ for the upper side, with $\mathrm{x} / \mathrm{c}=$ 0 being the leading edge. The uncorrected angle of attack is $\alpha=3 \mathrm{deg}$. The largest fluctuations in pressure are found at about $45 \%$ of the aerofoil chord, which correspond to the time averaged position of the shock on the upper surface. Outside the range in which the shock periodically moves, the flow field is relatively steady as shown by the low level of fluctuations. Qualitatively, the URANS solution provides a reasonable prediction of the shock-buffet at these flow conditions. 


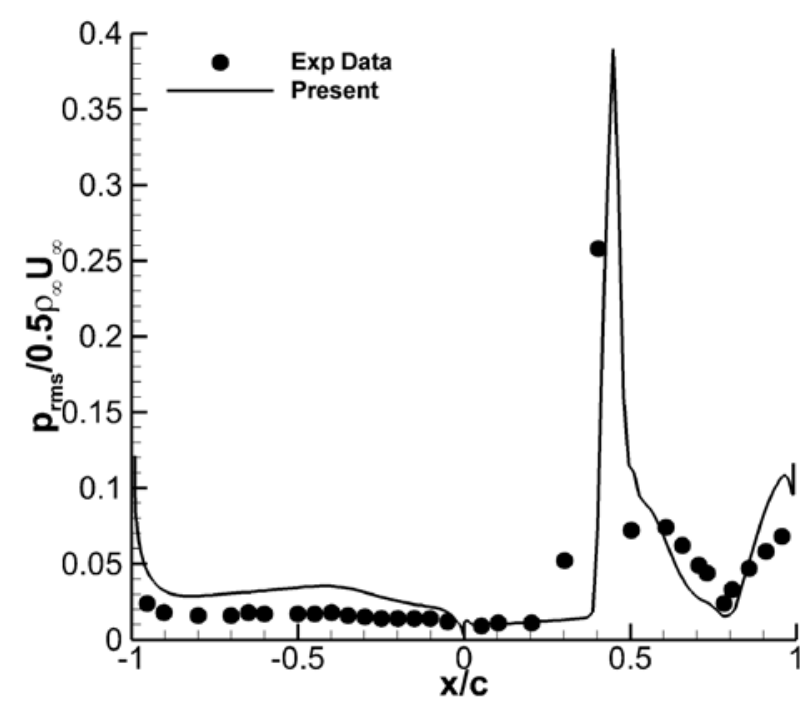

Figure 3 Root mean square constant head pressure fluctuations for the RA16SC1 aerofoil $\left(\alpha=3 \mathrm{deg}, M_{\infty}=0.732, R e=4.2 \times\right.$ $\left.10^{6}\right)$.

\section{Efficient identification of buffet envelope}

The second phase of this work concerns the identification of the buffet envelope of the reference equivalent wings at a fixed Mach number, $M_{\infty}^{\prime}=0.723$. The buffet envelope is presented within the space of the (corrected) angle of attack, $\alpha^{\prime}$, and the wing sweep angle, $\Lambda$. For a given wing sweep angle, buffet is identified as the angle of attack at which self-sustained, harmonic fluctuations in the lift coefficient reach \pm 0.001 . The exploration of the $\alpha^{\prime}-\Lambda$ plane was carried out using the infinite sweptwing flow solver on a purely 2D grid stencil, and results are summarised in Figure 4. The influence of the wing sweep angle within the context of a purely 2D grid stencil is dealt with imposing appropriate boundary conditions at the far-field (Franciolini et al., 2017). In practice, the search was conducted, for each sweep angle, increasing the angle of attack between 1 and $7 \mathrm{deg}$, at increments of 1 deg initially. Once the buffet onset (and offset) was recorded to occur at two neighbouring values of the angle of attack, one additional calculation was performed at the mean value of these two angles of attack. The resulting uncertainty in the prediction of the buffet envelope is reduced to less than $0.5 \mathrm{deg}$, as indicated by the uncertain band of Figure 4 . The prediction of the buffet envelope for the proposed design space required a total of 52 computations involving the solution of the infinite swept-wing URANS equations on a 2D grid stencil (27 thousand mesh elements).

Computed results in this work, in conjunction with wind tunnel experimental results presented in (Dandois, 2016), allows one to build a notional understanding of the influence that the wing sweep angle, $\Lambda$, has on the buffet onset (and offset) curve. Figure 4 shows that increasing $\Lambda$ tends to move the buffet onset curve toward higher angles of attack but has a minimal influence on the buffet offset curve. For the largest value of $\Lambda$, buffet was not recorded to occur at any angles of attack. The reason for this 
behaviour is found investigating the flow field for increasing $\Lambda$ for a given $\alpha^{\prime}$. Figure 5 shows the distributions of the time averaged pressure coefficient related to the four cases indicated by the blue arrow in Figure $4\left(\alpha^{\prime}=3 \mathrm{deg}\right)$. For the transonic speed tested at which buffet is due to shock-induced separation, increasing $\Lambda$ increases the 3D character of the shock, weakening the shock intensity and moving the shock front upstream toward the leading edge. In turn, the size of the shock-induced boundary layer separation is greatly reduced from the initial extent covering the aft $50 \%$ of the aerofoil chord to virtually disappearing for $\Lambda=30 \mathrm{deg}$.

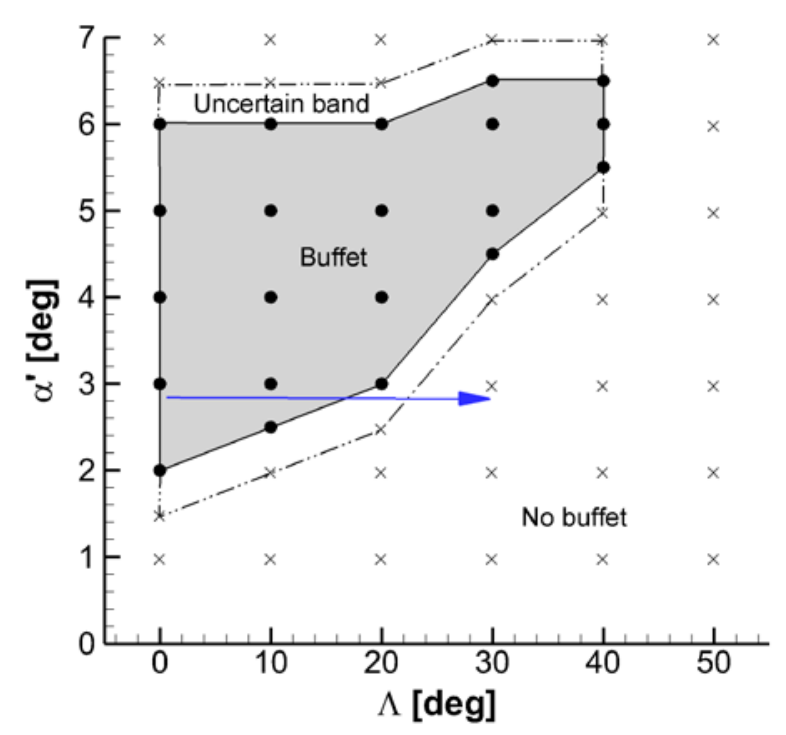

Figure 4 Prediction of buffet envelope of the reference equivalent wings using a purely $2 \mathrm{D}$ grid stencil $\left(M_{\infty}^{\prime}=0.723, R e=\right.$

\section{$\left.4.2 \times 10^{6}\right)$}
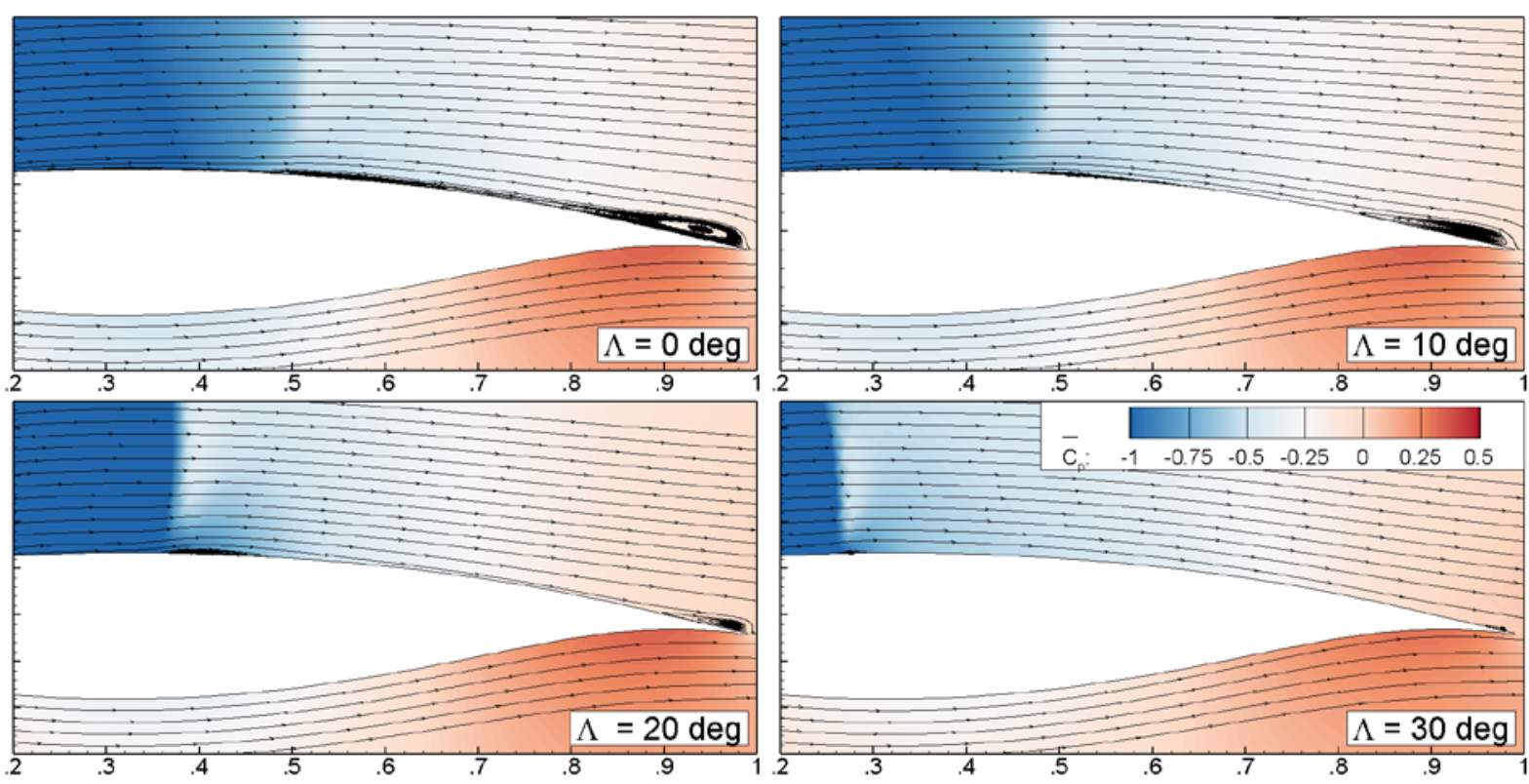

Figure 5 Dependence of time averaged pressure coefficient on sweep angle computed on a purely 2D grid stencil at $\alpha^{\prime}=3 \mathrm{deg}$

$$
\left(M_{\infty}^{\prime}=0.723, R e=4.2 \times 10^{6}\right)
$$


Having good estimates of the unsteady load characteristics at fully developed buffeting conditions is critical to carry out an appropriate structural design against fatigue failure. For fatigue design, statistics of the loads are needed, as shown in Figure 6. The time history of the lift coefficient is represented by the time averaged value, $\bar{C}_{L}$, the amplitude of self-sustained, harmonic fluctuations around $\bar{C}_{L}$, denoted $\Delta C_{L}$, and the reduced frequency of buffeting. Figure 6 conveys the dependence of the load characteristics on $\Lambda$ for two values of $\alpha^{\prime}$. For values of the sweep angle at which buffeting conditions are persistent, $\bar{C}_{L}$ and $\Delta C_{L}$ fall at a small rate for increasing $\Lambda$. This can be observed for sweep angles up to 15 deg for $\alpha^{\prime}=3$ deg, and up to 35 deg for $\alpha^{\prime}=5$ deg. Nearer the buffet onset curve, $\Delta C_{L}$ drops sharply to zero with a concurrent, but localized, increase in $\bar{C}_{L}$. The reduced frequency of buffeting shows a monotonic decreasing trend with the sweep angle, which becomes more accentuated near or at the buffet curve onset.

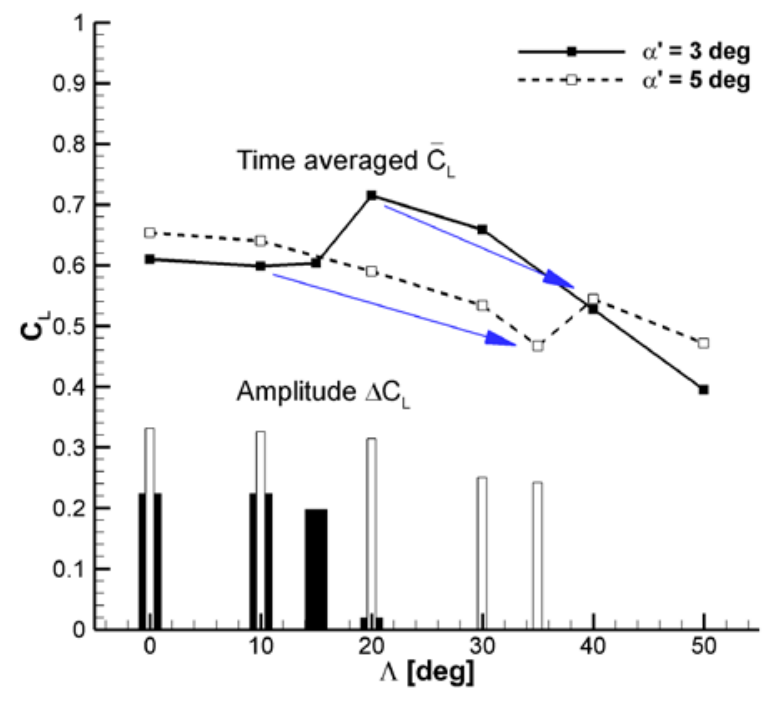

(a) Time averaged value and amplitude

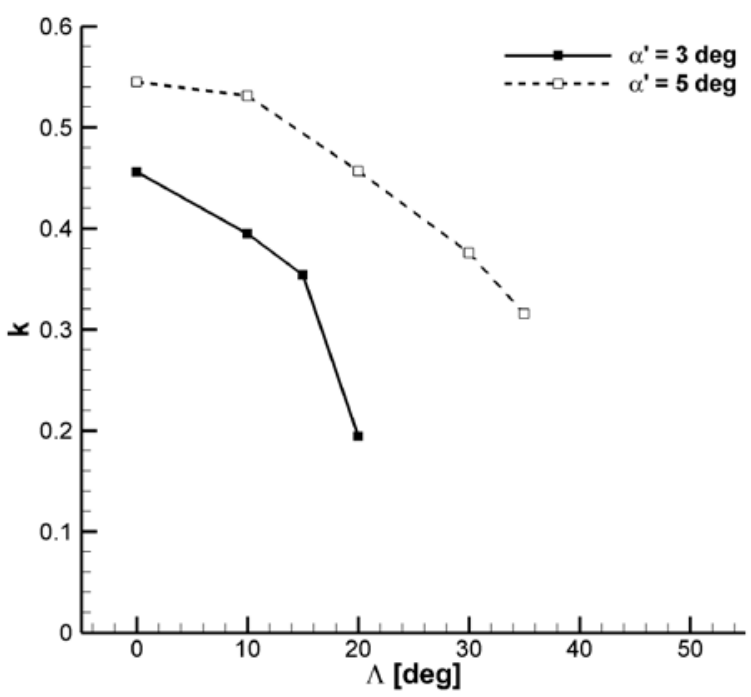

(b) Reduced frequency

Figure 6 Dependence of unsteady lift characteristics on sweep angle computed on a purely 2D grid stencil $\left(M_{\infty}^{\prime}=\mathbf{0 . 7 2 3}, R e=\right.$

$$
\left.4.2 \times 10^{6}\right)
$$

\section{Verification of the buffet envelope}

The third phase of this work aims at improving the accuracy of the buffet envelope, previously identified using the infinite-swept wing flow solver on a purely 2D grid stencil (Figure 4), at a minimal additional computational cost. To achieve higher prediction accuracy, the 3D URANS equations are solved around a 3D grid of the reference equivalent wings (about 6 million mesh elements). To limit the computational cost that would otherwise become prohibitive for a design exploration, the buffet envelope obtained on a purely 2D grid stencil is used as a starting point to deploy, at strategic locations, the 3D URANS calculations. Referring to Figure 7, the following procedure was implemented. For the buffet onset curve, a 3D URANS calculation is initially run, for a given $\Lambda$, at the angle of attack at which buffet onset was recorded on a purely $2 \mathrm{D}$ grid stencil. If no buffet is found on the fully 3D grid, the subsequent calculation is run at an angle of attack incremented by 0.5 deg from the previous 
value. An example of the iterative process, initialised from the approximate value of buffet onset, is indicated by an arrow in Figure 7 for $\Lambda=20$ deg. A similar procedure was followed for detecting the buffet offset curve. In total, 21 computations involving the 3D URANS equations were run to obtain prediction in buffet envelope to an accuracy of $\pm 0.5 \mathrm{deg}$.

Figure 7 compares the buffet envelope obtained using the purely 2D grid stencil (black) and the fully 3D grids (red). The influence of the sweep angle on the buffet envelope, already discussed, is confirmed here by the expensive URANS calculations run around the 6 million mesh elements. Quantitatively, one may observe some discrepancies (varying up to 1 deg) regarding the buffet onset/offset angle of attack between the two sources of aerodynamic predictions. Taking as reference the buffet from the 3D URANS calculations, the approximation error implicitly introduced when using the buffet envelope from the purely 2D grid stencil calculations is less than 1 deg overall. This demonstrates that the approach presented herein is valid to leverage on an efficient and error-bounded buffet envelope for design exploration using a lower fidelity model, which may be further refined by targeting the higher fidelity model at specific locations of the design space.

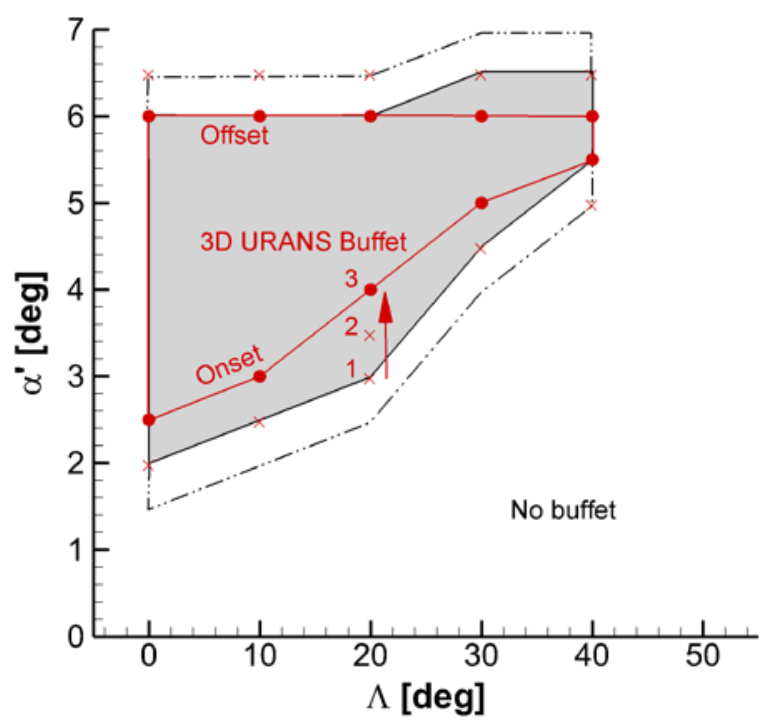

Figure 7 Prediction of buffet envelope of the reference equivalent wings using a fully 3D grid compared to that using a purely 2D

$$
\text { grid stencil }\left(M_{\infty}^{\prime}=0.723, \operatorname{Re}=4.2 \times 10^{6}\right)
$$

\section{Computational costs}

All URANS analyses were carried out for 4,000 physical time steps, allowing sufficient time for buffet to fully develop as a selfsustained, harmonic excitation. In terms of computational time, one single infinite swept-wing URANS calculation was obtained in about 45 CPU hours, which increased to about 20,000 CPU hours for one single 3D URANS calculation.

To obtain an indicative understanding of the advantages brought forward by the presented approach, two scenarios are investigated. The first scenario concerns the identification of the buffet envelope using solely 3D URANS calculations. In total, 
52 calculations would be needed to cover the 2D design space (seven values of the angle of attack and six values of the wing sweep angle) and to reduce the uncertainty in the angle of attack at which buffet onset/offset is detected to \pm 0.5 deg. This amounts to more than one million CPU hours of computing. In the second scenario, previous knowledge about the buffet envelope obtained using the infinite-swept wing solver allows limiting the number of expensive 3D URANS calculations. This scenario corresponds to the approach illustrated in Figure 7, and required a total of about 422 thousand CPU hours (52 runs on a purely $2 \mathrm{D}$ grid stencil and 21 runs on a fully $3 \mathrm{D}$ grid).

Based on the above paragraphs, one may wish to obtain rapidly a buffet envelope using a purely 2D grid stencil. In the event that results with a bounded uncertainty of \pm 1.0 deg are acceptable, the task is completed in about 2,340 CPU hours. However, if a requirement exists to halve the uncertainty in the buffet envelope, CPU resources to be allocated for the task will increase by a factor close to 200. With the access to the most efficient algorithms and best practice, this is still 2.5 times faster than employing a brute force approach in combination with fully 3D URANS calculations, but without any reduction in uncertainty.

\section{Lessons learned on numerical set-up}

Although the literature on computational investigations of buffet is substantial, there is a lack of a truthful assessment of the challenges to be faced to guarantee consistency, repeatability and robustness of the computed results. We have observed that the strategy employed to generate a 3D grid has a strong impact on buffet appearance.

- Even though the dimensionless wall distance equal to unity should be sufficient to detect buffeting when using the original SA turbulence model (Spalart and Allmaras, 1994), we could not capture this phenomenon when the average $y^{+}$was about one. We investigated further cases by reducing the minimal distance from the wall of the RA16SC1 aerofoil so that the average $y^{+}$was less than 0.5 , i.e. the first layer on the wall is placed at $4 \times 10^{-6}$ for a chord of one.

- A further decrease of the first layer on the wall did not have any effect on the numerical solution.

- The span-wise refinement was also found to have a strong impact on computed results. Based on background studies on the reference equivalent wings, our recommendation is to have a span-wise cell size smaller than $4 \times 10^{-2}$ for a chord of one.

- Furthermore, it was observed, that buffet can only be captured using the first order Roe scheme for SA equation.

\section{Conclusions}

The paper presented a numerical method based on computational fluid dynamics that allows discovering the buffet envelope of reference equivalent wings at the equivalent cost of several two-dimensional unsteady analyses. To permit the use of the 
proposed method as a practical design tool in the conceptual/preliminary aircraft design phases, the method offers the ability to gauge the sensitivity of buffet on primary design variables, such as wing sweep angle and chord to thickness ratio.

The efficient identification of the buffet envelope, defined within the space of angle of attack and wing sweep angle, benefits from a novel implementation of the infinite-swept wing unsteady flow equations. It is the first time that this type of specialised flow solver is applied successfully for buffet calculations. First, a preliminary study was carried out to validate the numerical settings for a canonical aerofoil problem, and then three-dimensional grids were carefully designed and generated. The buffet envelope, related to solving the unsteady, three-dimensional Reynolds-averaged Navier-Stokes equations, was taken as the reference. Within a realistic environment, one often struggles with the limited availability of computational resources or wallclock time. In this respect, the proposed method delivers the required trade-off between accuracy, or uncertainty in the predictions, and CPU time needs. In the event that an uncertainty of \pm 1.0 deg in the angle of attack at which buffet onset (offset) occurs, for a given wing sweep angle, is admissible, the corresponding buffet envelope is obtained in a little more than two thousand CPU hours. If there is a requirement to halve the uncertainty in predictions, CPU resources increase by a factor close to 200. This sharp increase in CPU time reflects the need to augment many infinite-swept wing calculations with few threedimensional flow equations, which drive the overall cost up.

\section{References}

Badcock K.J., Timme S. and Marques S., Khodaparast H., Prandina M., Mottershead J.E., Swift A., Da Ronch A. and Woodgate M.A. (2011). Transonic aeroelastic simulation for instability searches and uncertainty analysis. Progress in Aerospace Sciences, 47(5): 392-423. https://doi.org/10.1016/j.paerosci.2011.05.002.

Benoit B. and Legrain I. (1987). Buffeting Prediction for Transport Aircraft Applications Based on Unsteady Pressure Measurements. 5th Applied Aerodynamics Conference, AIAA Paper 1987-2356.

Bérard, A. and Isikveren, A. T. (2009). Conceptual Design Prediction of the Buffet Envelope of Transport Aircraft. Journal of Aircraft, 46(5), 1593-1606. https://doi.org/10.2514/1.41367

Coustols E. and Schaeffer N., T.M., F.P.C. (2003). Unsteady Reynolds-averaged Navier-Stokes computations of shock induced oscillations over two-dimensional rigid airfoils. TSFP Conference Series.

Dandois J. (2016). Experimental study of transonic buffet phenomenon on a 3D swept wing. Physics of Fluids, 28(1): 1-22. Drofelnik J. and Da Ronch A. (2017). 2.5D+ User Guide. DLR-Tau Documentation.

Franciolini, M. and Da Ronch A., D.J., R.D, C.A. (2017). Efficient Infinite-swept Wing Solver for Steady and Unsteady Compressible Flows. Aerosp. Sci. Technol., 72: 217-229. 
Girodroux-Lavigne P and Le Balleur J.C. (1987). Unsteady viscous inviscid interaction method and computation on buffeting over airfoils. Proceedings of Joint IMA/SMAI Conference, Computational Methods in Aeronautical Fluid Dynamics. University of Reading. Berlin: Springer.

Girodroux-Lavigne P and Le Balleur J.C. (1988). Time consistent computation of transonic buffet over airfoils. Proceedings of 16th Congress of the International Council of the Aeronautical Sciences, ICAS, 88: 779-787.

Iovnovich, M. and Raveh, D. E. (2012). Reynolds-Averaged Navier-Stokes Study of the Shock-Buffet Instability Mechanism. AIAA Journal, 4: 880-890.

Iovnovich, M. and Raveh, D. E. (2015). Numerical Study of Shock Buffet on Three-Dimensional Wings. AIAA Journal, 2: 449-463. Jameson A. and Schmidt W., T.E. (1981) Numerical solutions of the Euler equations by finite volume methods using Runge-Kutta time-stepping schemes. 14th Fluid and Plasma Dynamics Conference.

Lee, B. H. K. (2001). Self-Sustained Shock Oscillations on Airfoils at Transonic Speeds. Progress in Aerospace Sciences, 2: 147196.

Schwamborn D. and Gerhold T., H.R. (2006). The DLR TAU-code: recent applications in research and industry. Proceedings of the European Conference on Computational Fluid Dynamics (ECCOMAS).

Spalart P. R. and Allmaras S. R. (1994). A one-equation turbulent model for aerodynamic flows. La Recherche Aérospatiale, 1: $5-21$.

Thiery, M. and Coustols, E. (2005).. Flow, Turbulence and Combustion, 4: 331-354.

Zhong B. and Scheurich F., T.V., D.D. Turbulent Flow Simulations Around a Multi-Element Airfoil Using URANS, DES and ILES Approaches. 19th AIAA Computational Fluid Dynamics, AIAA 2009-3799.

\section{Acknowledgments}

Da Ronch and Drofelnik gratefully acknowledge the financial support from the Engineering and Physical Sciences Research Council (grant number: EP/P006795/1).

Data supporting this study are openly available from the University of Southampton repository at http://dx.doi.org/10.5258/SOTON/xxxxx. 